\title{
A STUDY ON DERMATOPHYTOSES IN A TERTIARY CARE HOSPITAL OF WEST BENGAL
}

\author{
Sanjit Kumar Patra1 ${ }^{1}$ Sanjeev Das ${ }^{2}$, Jayashree Konar ${ }^{3}$ \\ ${ }^{1}$ Associate Professor and HOD, Department of Microbiology, College of Medicine and Sagore Dutta Hospital, Kamarhati, Kolkata. \\ ${ }^{2}$ Assistant Professor, Department of Microbiology, R.G. Kar Medical College and Hospital, Kolkata. \\ ${ }^{3}$ Demonstrator, Department of Microbiology, Nil Ratan Sircar Medical College and Hospital, Kolkata.
}

\section{ABSTRACT}

\section{BACKGROUND}

Dermatophytoses refer to superficial fungal infection of keratinized tissues- skin, hair and nail caused by keratinophilic dermatophytes. According to worldwide scientific observations, dermatophytoses are the most common of the superficial fungal infections, especially in tropics and subtropics due to high humidity.

The study was performed to assess the occurrence of different clinical profile of dermatophytic infection in a tertiary care hospital of West Bengal and to identify the species of various dermatophytes.

\section{MATERIALS AND METHODS}

This is a descriptive study. A total of 85 clinically suspected cases of dermatophytoses, attending a tertiary care hospital in Kolkata were selected for the study. Direct microscopy in $10 \%$ potassium hydroxide (KOH) for skin and hair and $20 \% \mathrm{KOH}$ for nail were performed. Culture was done in Sabouraud Dextrose Agar (SDA) and Sabouraud Dextrose Agar with chloramphenicol and cycloheximide (SDCCA).

\section{RESULTS}

Out of 85 clinically suspected cases, 79 presented with tinea cruris, 4 cases presented with onychomycosis and rest 2 cases presented with tinea capitis. Majority of the cases (67\%) were reported from low income group (Below Poverty Line card holder); 67 cases were positive on direct $\mathrm{KOH}$ mount and 29 were culture positive. Amongst these 29 culture positive cases, in 23 cases both $\mathrm{KOH}$ and culture were positive (79\%) and remaining 6 cases were negative on direct microscopy showed culture positivity. Trichophyton rubrum was the predominant species isolated (82\%) in all clinical types followed by Trichophyton mentagrophytes (18\%).

\section{CONCLUSION}

Culturing the fungus for identification of the species is not always possible due to low sensitivity.

\section{KEYWORDS}

Dermatophyte, Tinea cruris, Trichophyton rubrum.

HOW TO CITE THIS ARTICLE: Patra SK, Das S, Konar J. A study on dermatophytoses in a tertiary care hospital of West Bengal. J. Evolution Med. Dent. Sci. 2017;6(76):5415-5418, DOI: 10.14260/Jemds/2017/1174

\section{BACKGROUND}

The cutaneous mycoses are caused by dermatophytes, which infects keratinised tissues including skin, hair follicles and nails. Dermatophytoses refer to superficial fungal infection of keratinised tissues- skin, hair and nail caused by keratinophilic dermatophytes. These dermatophytic fungi invade the epidermis and elicit an inflammatory reaction including redness and pruritus. Typically, it results in a red, itchy, scaly, circular rash. Hair loss may occur in the affected areas. It is commonly known as ringworm or tinea infection. Description of ringworm had been reported, which date back to ancient history. Dermatophytic infections are designated according to the anatomic location of the lesions- e.g. tinea corporis (the trunk, shoulders or limbs), tinea cruris (the warm moist areas of the groin, perianal and perineal areas),

Financial or Other, Competing Interest: None.

Submission 07-08-2017, Peer Review 08-09-2017,

Acceptance 13-09-2017, Published 21-09-2017.

Corresponding Author:

Dr. Sanjit Kumar Patra

IB 10/1, Sector-3,

Salt Lake City, Kolkata-106,

West Bengal.

E-mail: sanjitkumarpatra@gmail.com

DOI: $10.14260 /$ jemds $/ 2017 / 1174$

\section{(c) $($ ) $९$}

tinea faciei (the non-hairy areas of the face), tinea pedis (the feet), tinea unguium (the nails) and tinea capitis (the scalp). ${ }^{1}$ Causative fungi are different species of Trichophyton, Microsporum and Epidermophyton. Microsporum spp. does not infect nail, whereas Epidermophyton spp. does not infect hair. ${ }^{2,3}$ Members of these genera can be divided into three groups according to their natural reservoir and potential for infection: anthropophilic, zoophilic and geophilic organisms. Tinea is common worldwide. It is estimated that more than 8 million office visits to primary care physicians are made annually for tinea-related symptoms. Dermatophytic fungi release proteolytic enzymes and keratinases into the skin. These exocellular enzymes release nutrients and facilitate dissemination through the stratum corneum. A specific host immune response is directed against the organisms. Any dermatophyte can cause tinea corporis, which is commonly called "ringworm" because of the typical appearance of lesions: annular scaly patches with raised, erythematous vesicular borders and central clearing. Tinea faciei like tinea corporis can be caused by any dermatophyte. T. rubrum and E. floccosum are common causes of tinea cruris; similar lesions can be caused by Candida infection. Tinea pedis, one of the most common clinical dermatophytic infection, usually presents with interdigital cracking, scaling and maceration. Hyperkeratosis and peeling of the soles of the feet are 
common with a scaly red "moccasin like" appearance in chronic cases. The most common cause of tinea pedis is $T$. rubrum. Clinical lesions similar to those of tinea pedis can be caused by non-dermatophytic fungi, yeasts and bacteria. Tinea unguium is caused by T. rubrum, T. mentagrophytes and $E$. floccosum. The term onychomycosis encompasses nail infections due to either dermatophytes or non-dermatophytic fungi. Dermatophytes cause $80 \%-90 \%$ of cases of onychomycosis. The prevalence of these infections is $\sim 2 \%$ among young adults and increases to $20 \%$ among individuals of 40 - 60 years of age. Onychomycosis occurs in diabetic patients at the same rate as in the general population, but poses a greater risk of bacterial superinfection in diabetes. Tinea capitis is a common dermatophytic disease of children, but is relatively rare among adults. The clinical presentation may vary from a diffuse scaly scalp to scattered areas of scale with or without alopecia. Hair may break off at the scalp ("Black-dot ringworm"). Pruritus is not a constant symptom. Inflammatory responses may be minimal or severe with the formation of a kerion. ${ }^{4}$

Symptoms begin four to fourteen days after exposure. Multiple areas can be affected at a given time. Risk factors include sharing personal items, contact sports such as wrestling, excessive sweating and contact with animals, obesity and poor hygiene. Immunocompromised states like diabetes, intake of immunosuppressant drugs, e.g. steroids also predispose tinea. Moreover, use of steroids and other immunosuppressant drugs may cause morphological alteration of typical clinical manifestations of dermatophytes. It may lead to several diagnostic dilemmas.5,6 Ringworm can spread from other animals or between people. Diagnosis is often based on the clinical presentations. Prevention is by keeping the skin dry, not walking barefoot in public and not sharing personal items. ${ }^{7,8}$ Upto $20 \%$ of the population may be infected by ringworm at any given time. ${ }^{9}$ Infections of the groin are more common in males, while infections of the scalp and body occur equally in both sexes. Infections of the scalp are most common in children, while infections of the groin are most common in the elderly.

According to worldwide scientific observations, dermatophytoses are the most common of the superficial fungal infections, especially in tropics and subtropics due to high humidity. Dermatological manifestations of psoriasis, contact dermatitis, etc. are the differential diagnoses. Identification of dermatophytes are based on use of Wood's lamp, direct microscopy of $\mathrm{KOH}$ mounted skin, hair or nail sample as applicable and culture techniques. Species confirmation is done by genotyping. Some skin lesions have distinctive characteristics that allow a presumptive diagnosis and topical therapy is often initiated solely on the basis of the lesion's appearance. However, the ease of obtaining specimens for microscopic examination and culture should encourage definitive diagnosis. Scrapings of skin lesions can be examined as a wet mount preparation with a drop of $\mathrm{KOH}$ used to dissolve keratin and debris. Samples for fungal cultures should be obtained from patients whose history, physical examination and $\mathrm{KOH}$-treated specimens are inconclusive with regard to the diagnosis of dermatophytic infection. It is recommended that a definitive diagnosis be established in patients before systemic antifungal agents are administered. 10
Most tinea infections can be treated with topical agents alone. Many such antifungal agents are widely available as both prescription and over-the-counter products. Topical imidazoles (e.g. clotrimazole, miconazole, econazole and ketoconazole) are generally well tolerated and efficacious when used twice daily for at least 2 weeks. The allylamines, including terbinafine and naftifine (available in $1 \%$ creams or $1 \%$ solutions), provide cure rates of $\geq 75 \%$ and require only once-daily application for shorter periods. Tolnaftate powder is best suited for prevention of tinea pedis. Systemic therapy is indicated for patients who are resistant to topical therapy; for those who have infections involving the scalp or bearded areas, who have hyperkeratotic areas on the palms or soles, or who have widespread disease; and for immunocompromised individuals. Once-daily itraconazole (200 mg), terbinafine $(250 \mathrm{mg}$ ) and griseofulvin (500 $\mathrm{mg}$ of the microcrystalline formulation or $375 \mathrm{mg}$ of the ultramicrocrystalline formulation) has proved effective. Treatment should be administered until lesions resolve. For patients with nail disease, itraconazole $(200 \mathrm{mg} / \mathrm{d})$ or terbinafine $(250 \mathrm{mg} / \mathrm{d})$ is preferred. The duration of therapy is 2 - 3 months for fingernails and $4-6$ months for toenails. Pulse therapy with itraconazole and terbinafine is an option. Relapse of nail disease is common. ${ }^{11}$ According to several scientific evidences, antifungal resistance is the most important cause for the treatment failure of ring worm. To understand the reality, it is essential for us to consider few facts about antifungal resistance, the occurrence of which has to be considered independently for each antifungal class and for each fungal genus. Antifungal resistance is broadly classified into microbiological resistance and clinical resistance. Microbiological resistance refers to nonsusceptibility of a fungus to an antifungal agent as determined by in vitro susceptibility testing, in which the minimum inhibitory concentration (MIC) exceeds the susceptibility breakpoint for that organism. Microbiological resistance can be primary or secondary resistance. Standard antifungal susceptibility tests (AST) for the moulds are generally cumbersome to perform than the antibacterial susceptibility tests. Hence, they are best performed by reference laboratories. At present, standard guidelines have been proposed by the Clinical Laboratory Standards Institute (CLSI), United States and European Committee on Antibiotic Susceptibility Testing (EUCAST) for in vitro antifungal susceptibility testing of dermatophytes. Broth microdilution method of AST recommended by CLSI and EUCAST is presently accepted as the standard method of testing. MIC is defined as the lowest concentration of an antifungal agent that will inhibit the visible growth of microorganism. Although, antifungal resistance is usually correlated with increased MIC, MIC values do not always correlate with clinical response to antifungal drugs. The discordance between the in vivo and in vitro resistance in several fungi has been illustrated by the "90 - 60 rule," which states that infections due to susceptible strains respond to appropriate therapy in $90 \%$ of cases, whereas infections due to resistant strains respond in approximately $60 \%$ of patients. Breakpoints also termed as interpretive criteria are used to denote susceptibility and resistance to antifungal agents as the outcome of AST. They are categorised as susceptible, 
intermediate and resistant. However, till now the breakpoints have not been defined for the dermatophytes due to lack of data on the clinical correlation, pharmacokinetic/ pharmacodynamic studies or epidemiological cut-off MIC values. Hence, experts opine that it is logical not to use the term- 'resistant' in the absence of these definitive criteria for dermatophytes. ${ }^{2}$

As there is a dire need for well-designed studies as well as more solid evidence for various issues pertaining to the dermatophytoses scenario in India, ${ }^{12,13}$ the present study aims to throw a light in this field.

\section{Objectives}

1. Assessment of occurrence of different clinical profile of dermatophytic infection in a tertiary care hospital of West Bengal.

2. Identification of the species of various dermatophytes.

\section{MATERIALS AND METHODS}

This is a descriptive study. A total of 85 clinically suspected cases attending a tertiary care hospital in Kolkata were selected for the study. Known immunocompromised cases and patients partially or fully treated with antifungal were excluded from the study.

Sample collection was done by skin scraping, hair cutting and plucking and nail scraping and clipping.

Collection and processing of the sample- Samples were collected from affected lesions. Whenever the patients presented with lesions at clinically different sites, samples were collected from all those sites and each of these were processed and examined individually. Collection of samples from skin- The affected area was swabbed with $70 \%$ alcohol and the active edge of lesion scraped with a flame sterilised blunt scalpel. The scrapings were collected from active margin of lesion without injuring the skin surfaces. Affected nails were swabbed with $70 \%$ alcohol, after which the nails were scraped deeply enough to obtain recently affected nail tissue. Nail clippings were also collected in addition to nail scrapings from the lesions whenever it is feasible. From the scalp, the above-mentioned procedure for skin scrapings was followed, in addition few affected hairs were also epilated and collected with a pair of sterile forceps. Care was taken to collect the basal portion of hair (hair stub) as the fungus is usually found in this area. If it was not possible due to hair fragility as in black dot in Tinea capitis, a sterile scalpel was used to scrape scales surrounding the hair root and excavate the remaining portions of the hair root. The nail clippings and hair samples were cut into small fragments. Direct microscopy in $10 \%$ potassium hydroxide $(\mathrm{KOH})$ for skin and hair and $20 \% \mathrm{KOH}$ for nails were performed. Hyaline, refractile, septate and branched hyphae were found in direct microscopy.

Culture was done in Sabouraud Dextrose Agar (SDA) and Sabouraud Dextrose Agar with chloramphenicol and cycloheximide (SDCCA) at $25^{\circ} \mathrm{C}$ maximum upto 4 weeks. To prepare SDCCA media, chloramphenicol $(0.05 \mathrm{mg} / \mathrm{mL})$ and cycloheximide $(0.5 \mathrm{mg} / \mathrm{mL})$ were added to SDA media. Any visible growth on SDA or SDCCA was examined for colony morphology on surface (obverse) and pigmentation on the reverse. Microscopic examination of colony was done by doing a Lactophenol Cotton Blue (LPCB) mount to examine the hyphal structure, different vegetative structures formed by hyphal modifications, various reproductive structures like microconidia, macroconidia and chlamydoconidia. Urea hydrolysis test was performed for speciation.

Data compilation and analysis were done as per standard statistical techniques. There was no violation of copyright or conflict of interest.

\section{RESULTS}

Out of 85 clinically suspected cases, 79 presented with tinea cruris, 4 cases presented with onychomycosis and rest 2 cases presented with tinea capitis.

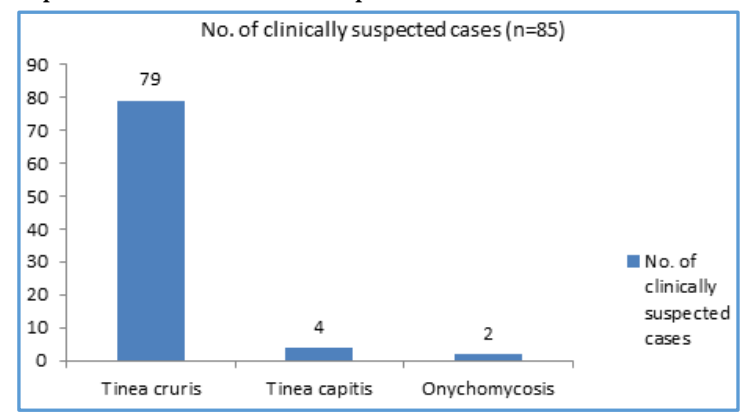

Figure 1. Bar showing Distribution of different Clinical Pattern of Dermatophytoses

Majority of the cases (67\%) were reported from low income group (Below Poverty Line card holder).

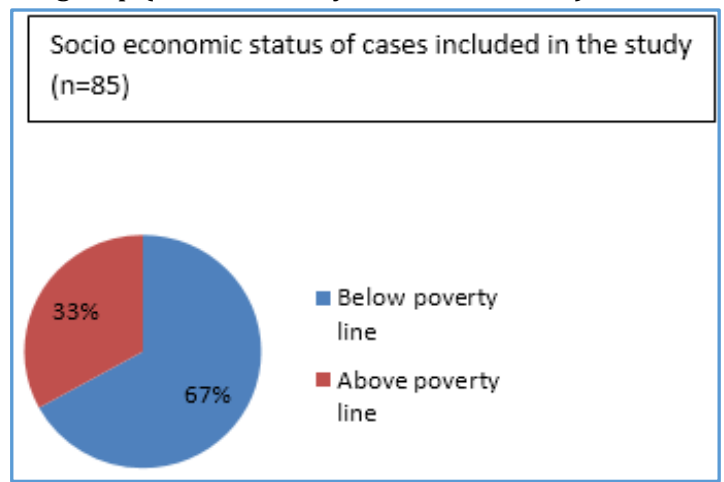

Figure 2. Pie showing Distribution of Clinically Suspected Cases according to their Socio-Economical Status

67 cases were positive on direct $\mathrm{KOH}$ mount and 29 were culture positive. Amongst these 29 culture positive cases, in 23 cases both $\mathrm{KOH}$ and culture were positive $(79 \%)$ and remaining 6 cases were negative on direct microscopy which showed culture positivity.

Trichophyton rubrum was most commonly isolated (24 out of 29, i.e. $82 \%$ ) followed by Trichophyton mentagrophytes (5 out of 29 , i.e. $18 \%$ ).

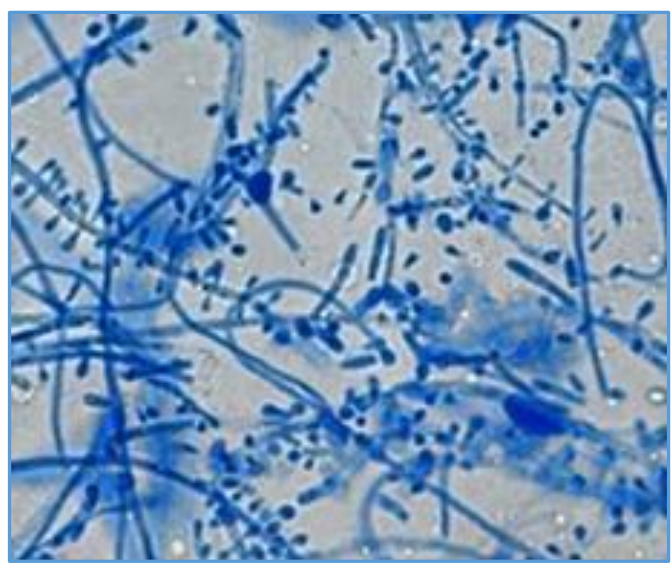

Figure 3. Trichophyton rubrum (LPCB Mount) 


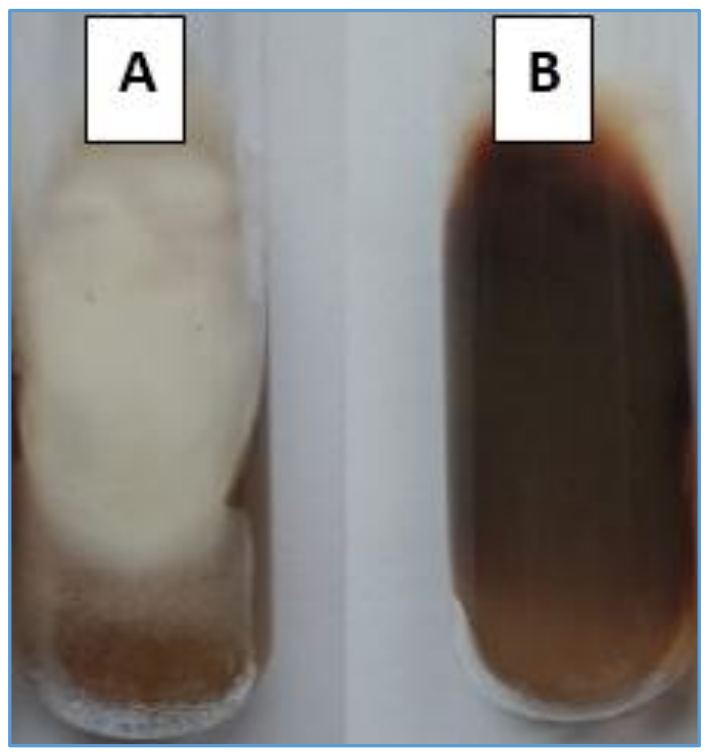

Figure 4. Trichophyton rubrum (SDA Media). A) Obverse Side, B) Reverse Side

\section{DISCUSSION}

In accordance to the findings of Grover WC et al,14 the present study reports various clinico-mycological profile caused by dermatophytoses such as tinea cruris, onychomycosis and tinea capitis.

In this present study, commonest clinico-mycological presentation was tinea cruris (92.9\%), whereas Ghosh et al ${ }^{15}$ has reported tinea unguium to be the commonest one. Majority of the cases (67\%) have been reported from the lower income group in accordance with the finding of Das et $\mathrm{al}^{1}$ and Ghosh et $\mathrm{al}^{15}$ The study conducted in eastern India shows that $57 \%$ of patients who came from urban background were compared to $43 \%$ from rural areas. ${ }^{15}$ In this present study, 67 cases were positive on direct $\mathrm{KOH}$ mount and 29 were culture positive. Amongst these 29 culture positive cases, 23 cases were both $\mathrm{KOH}$ and culture positive (79\%) and remaining 6 cases were negative on direct microscopy which showed culture positivity. Similar to our findings, Ghosh et al ${ }^{15}$ also reported lower culture positivity in their study. In this study, Trichophyton rubrum was the predominant species isolated (82\%) from all clinical types followed by Trichophyton mentagrophytes (18\%). As reported by Kannan et al, in their study dermatophytes were isolated in 53/80 (66.3\%) specimens and Trichophyton rubrum was the commonest isolate in skin samples $(17 / 24)$ of the patients. ${ }^{8}$ Das et $\mathrm{al}^{1}$ and Verma et $\mathrm{al}^{2}$ also reported Trichophyton rubrum as the commonest isolate in dermatophytoses.

\section{Further Scope}

Genotyping for species confirmation and antifungal susceptibility testing as per Clinical Laboratory Standard Institute (CLSI) guidelines or European Committee on Antibiotic Susceptibility Testing (EUCAST) will be the next approach of this study.

\section{CONCLUSION}

Trichophyton rubrum was the predominant species isolated $(82 \%)$ in all clinical types followed by Trichophyton mentagrophytes $(18 \%)$ in this study. Culturing the fungus for identification of the species is not always possible due to low sensitivity.

\section{ACKNOWLEDGEMENTS}

Authors cordially express their gratitude to all the faculty members and technical staff related to this study for their moral support and cooperation.

\section{REFERENCES}

[1] Das K, Basak S, Ray S. A study on superficial fungal infection from West Bengal: a brief report. J Life Sci 2009;1:51-5.

[2] Verma S, Madhu R. The great Indian epidemic of superficial dermatophytosis: an appraisal. Indian J Dermatol 2017;62(3):227-36.

[3] Narasimhalu CR, Kalyani M, Somendar S. A crosssectional, clinico-mycological research study of prevalence, aetiology, speciation and sensitivity of superficial fungal infection in Indian patients. J Clin Exp Dermatol Res 2016;7:324.

[4] LY R. Skin, hair, and nail fungal infections. Infect Dis Clin Pract 1998;10:117.

[5] Kumar S, Goyal A, Gupta YK. Abuse of topical corticosteroids in India: concerns and the way forward. J Pharmacol Pharmacother 2016;7(1):1-5.

[6] Lim SP, Smith AG. Tinea pseudoimbricata: tinea corporis in a renal transplant recipient mimicking the concentric rings of tinea imbricata. Clin Exp Dermatol 2003;28(3):332-3.

[7] Midgley G, Moore MK, Cook JC, et al. Mycology of nail disorders. Journal of the American Academy of Dermatology 1994;31(3):S68-74.

[8] Kannan P, Janaki C, Selvi GS. Prevalence of dermatophytes and other fungal agents isolated from clinical samples. Indian J Med Microbiol 2006;24(3):212-5.

[9] Nawal P, Patel S, Patel M, et al. A study of superficial mycosis in tertiary care hospital. Natl J Integr Res Med 2012;3:95-9.

[10] Milne LJR. Fungi. In: Mackie and McCartneyeds. Practical medical microbiology. 14th edn. Churchill Livingstone 2001:P 695.

[11] Chapman SW, Sullivan DC. Miscellaneous mycoses and algal infections (Chapter 110). Harrison's infectious diseases. 17th edn. Mc Graw Hill 2010:1030-8.

[12] Dogra S, Uprety S. The menace of chronic and recurrent dermatophytosis in India: is the problem deeper than we perceive? Indian Dermatol Online J 2016;7(2):73-6.

[13] Verma SB. Sales, status, prescriptions and regulatory problems with topical steroids in India. Indian J Dermatol Venereol Leprol 2014;80(3):201-3.

[14] Grover S, Roy P. Clinico-mycological profile of superficial mycosis in a hospital in North-East India. Med J Armed Forces India 2003;59(2):114-6.

[15] Ghosh RR, Ray R, Ghosh TK, et al. Clinico-mycological profile of dermatophytosis in a tertiary care hospital in West Bengal an Indian scenario. Int J Curr Microbiol App Sci 2014;3(9):655-66. 